\title{
Respiratory influences on cardiac responses during attention
}

\author{
MICHAEL N. CHEUNG and STEPHEN W. PORGES \\ University of Illinois, Champaign, Illinois 61820
}

\begin{abstract}
The effects of respiration inhibition on cardiac responses were investigated during two attentional tasks: reaction time and visual search. The responses were partitioned into two sequential components: a short-latency (reactive) acceleration and a longer latency (tonic) component characterized by directional and stabilization changes. The reactive cardiac response components were independent of changes in respiratory activity. Respiration inhibition during the tonic interval was related to both cardiac deceleration and stabilization in the reaction time task but not during visual search.
\end{abstract}

Research with cardiac responses has generally dealt with two theoretical constructs: the orienting reflex, a reflexive response to stimulus change, and sustained attention, a voluntary process. Cheung (Note 1) presented evidence suggesting that the cardiac response may be statistically partitioned via factor analytic techniques into multiple temporally distinct components. These various components may parallel the theoretical distinction between the orienting reflex and sustained attention. Components associated with the orienting reflex are generally short-latency directional changes and may be sensitive to the physical or signal qualities of the stimulus (Graham \& Clifton, 1966). Components associated with sustained attention consist of tonic directional changes and inhibition of variability (Porges, 1976) and may be influenced by the type of attentional effort involved.

Respiratory influence may denote another source of variance in the cardiac response. While it is easily demonstrated that respiratory activity may influence spontaneous cardiac activity (e.g., sinus arrhythmia; Guyton, 1971), few studies have investigated the relationship between respiratory activity and cardiac responses during attention-demanding tasks. Hart (1975) reported that respiratory amplitude at that time of stimulus onset may alter the short-latency components of cardiac response. It is possible that respiratory inhibition, a condition often associated with sustained attention (Porges, 1976), may modify the cardiac response during attention in

This investigation was supported, in part, by Research Scientist Development Award K02-MH-00054 from the National Institute of Mental Health and by National Institute of Educattion Grant NE-G-00-3-0013, both awarded to Stephen W. Porges. The research was completed while Michael N. Chueng was a trainee on U.S. Public Health Service Grant HD-00244. Requests for reprints should be addressed to Dr. Stephen W. Porges, Department of Psychology, University of Illinois, Champaign, Illinois 61820. a similar manner. Such respiratory influences on cardiac responses are likely to be mediated via vagal and brainstem mechanisms (Eckoldt \& Schubert, 1975; Katona \& Jih, 1975). Thus, it might be reasonable to expect the respiratory influences on the cardiac response to diminish during specific attentional tasks, which may require a substantial degree of cortical involvement, since cortical activities often have an inhibitory effect on subcortical processes.

The present experiment was an attempt to investigate cardiac responses during two tasks: reaction time and visual search. Based upon previous research (Walter \& Porges, 1976), the response was partitioned into two sequential components: a component of short-latency changes and a longer latency component characterized by directional and variability changes. Two hypotheses were tested: first, that the two cardiac response components may be differentially influenced by respiratory activities; and second, that the respiratory influence is attenuated during tasks which demand more cognitive mediation than simple sensorimotor performance.

\section{METHOD}

\section{Subjects}

Twelve male students from an introductory psychology course at the University of Illinois at Urbana-Champaign participated as subjects for course credit. All subjects had at least 20/20 vision, with or without the aid of corrective lenses. None reported existing neurological or physical disorders. All subjects completed the experimental sessions.

\footnotetext{
Apparatus

Stimuli for the various attention-demanding tasks were presented automatically via electronic timers and electromechanical programming devices. The ambient noise level in the laboratory was masked by a 60-dB white noise. Room temperature was maintained at approximately $70^{\circ} \mathrm{F}$. The onset of each task was signaled by a 75- $\mathrm{dB} 500-\mathrm{Hz}$ sine-wave tone with a $300-\mathrm{msec}$ rise time $^{1}$ in combination with either a yellow or a green $28-\mathrm{V}$ pilot light for the visual search and reaction time tasks, respectively. The respond signal for the reaction time task was a red pilot light.
} 


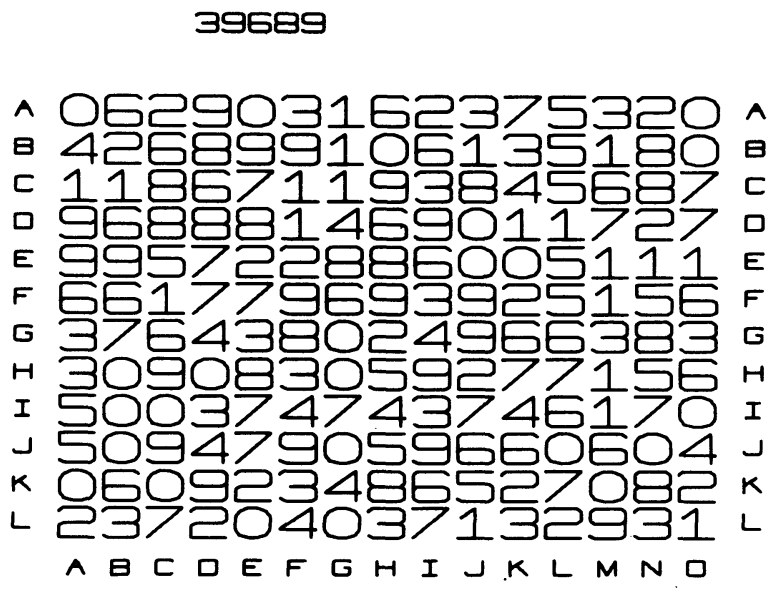

Figure 1. Sample matrix of random numbers in the visual search task.

The visual search stimulus consisted of a matrix of random numbers projected on a screen by a Kodak Model $650 \mathrm{H}$ Carousel slide projector and a Kodak dissolve unit.

The pilot lights were placed inside a $13 \times 10 \times 8 \mathrm{~cm}$ metallic enclosure and mounted behind a $48 \times 18 \mathrm{~cm}$ gray panel just below eye level on a standard laboratory instrument rack about $2 \mathrm{~m}$ in front of the subject. At the center of the panel was a $10 \times$ $8 \mathrm{~cm}$ frosted Plexiglas window, through which the lights were presented.

Matrices, as illustrated in Figure 1, were composed of 15 columns by 12 rows of uniform random numbers and were generated and plotted by an IBM 1800 computer. Slides of each matrix were projected through a window behind the subject onto a $80 \times 50 \mathrm{~cm}$ white screen mounted directly above the light presentation panel. The projected characters $(2.3 \times 2.8 \mathrm{~cm})$ were black on a white background, and were spaced $0.5 \mathrm{~cm}$ from each other. To each side and below the matrix were smaller $(1.2=$ $1.5 \mathrm{~cm}$ ) letters identifying the rows and columns of the matrix. Imbedded in the matrix in a horizontal, vertical, or diagonal direction was a sequence of five digits which was also presented above the matrix.

Electrocardiogram (EKG) and respiration were monitored continuously by a Beckman Type $\mathbf{R}$ Dynograph and recorded on an Ampex Model SP-500 FM tape recorder. EKG was recorded via Beckman 1-cm Ag-AgCl electrodes filled with Beckman electrode paste. Active electrodes were placed on the right collar bone and left leg. A ground electrode was placed on the right leg. Respiration was monitored by means of a $10-\mathrm{cm}$ mercury strain gauge placed above the abdomen. Resistance changes in the strain gauge occurred during breathing and were recorded with a time constant of $1 \mathrm{sec}$ on the polygraph.

\section{Procedure}

Subjects were asked to refrain from medication or strenuous exercises for at least $1 / 2 \mathrm{~h}$ prior to the experiment. They were tested individually in a $3 \times 5 \mathrm{~m}$ sound-attenuated room. The room was darkened except for indirect background illumination from a shaded, $40-\mathrm{W}$ tungsten lamp placed behind the subject.

At the beginning of the experimental session, the subject was seated in a classroom-type tubular desk-chair in the experimental room. After the electrodes and strain gauge were attached and the purpose of the physiological sensors explained, the subjects was left alone to relax while the equipment was calibrated. After the subject appeared to have adapted to the testing environment (indicated by slower and more regular cardiac patterns observed on the polygraph), a 2-min baseline of EKG and respira- tion was recorded. The experimenter then entered the room and instructed the subject about the required tasks.

The experiment consisted of at least 10 trials of warned reaction time and $\mathbf{1 0}$ trials of visual search tasks presented in a predetermined random order. At the beginning of each trial, $2 \mathrm{sec}$ of the 75-dB tone were presented simultaneously with the onset of a pilot light on the panel in front of the subject. If the light was green, the subject was instructed to attend and to press a handheld button as quickly as possible when it changed to red. If the light was yellow, the subject was instructed to wait until a matrix was projected on the screen (approximately $1.5 \mathrm{sec}$ rise time to peak illumination). When the subject identified the five-digit sequence in the matrix, he was instructed to press the hand-held button and the matrix would fade out. Subsequent to this button press, the subject was instructed to record the row and column of the matrix in which the numerical sequence began on a piece of paper.

The foreperiod for the reaction time trials and the length of the wait periods varied among 10,15 , and $20 \mathrm{sec}$. No time limit was imposed on the visual search trials. However, only trials that lasted $10 \mathrm{sec}$ or more were included in the analyses. Intertrial intervals varied among 25,30, and $35 \mathrm{sec}$. All stimulus presentations were preprogrammed. The experimental session continued until the subject had completed 10 trials of visual search, which lasted at least $10 \mathrm{sec}$ each. A typical session lasted between 30 and $50 \mathrm{~min}$.

\section{Quantification of Data}

To facilitate interpretation, since the wait subtask was always temporally linked to the matrix subtask, the data for these two subtasks as well as the reaction time task were analyzed in separate analyses of variance. Beat-by-beat heart periods were measured and respiration amplitude was digitized at each $\mathbf{R}$-wave starting $5 \mathrm{sec}$ prior to the onset of each trial and continuing through the first $10 \mathrm{sec}$ of each task condition, with an IBM 1800 computer. The $15 \mathrm{sec}$ of continuous data for each trial were converted into second-by-second weighted means for the heart period and the associated respiration. ${ }^{2}$

Each trial was divided into three 5 -sec sequential intervals: pretrial ( $5 \mathrm{sec}$ prior to trial onset), reactive (first $5 \mathrm{sec}$ of the trial), and tonic (second $5 \mathrm{sec}$ of the trial). Mean heart period, heart period $\log _{10}$ variance, and respiration amplitude $\log _{10}$, variance were computed for each of these intervals. Respiratory inhibition during the reactive and tonic intervals was computed as the respective change in respiration amplitude $\log _{10}$ variance during these intervals relative to the pretrial interval.

\section{RESULTS}

\section{Second-By-Second Cardiac Responses}

The second-by-second cardiac response pattern during each attentional task was evaluated by assessing heart period from the last pretrial second through the 10th second after task onset. Three 10 by 11 (Trail by Second) factorial analyses of variance (ANOVA) were performed. The second-by-second means for each of the three tasks are presented in Figure 2. For the reaction time task, the cardiac response $[F(10,110)=15.06, p<.01)]$ was triphasic, showing an initial slight deceleration followed by an acceleratory phase, followed in turn by cardiac deceleration. The visual search task was divided into wait and matrix subtasks. During the wait subtask, there was an initial slight deceleration which closely resembled the initial deceleration of the reaction 


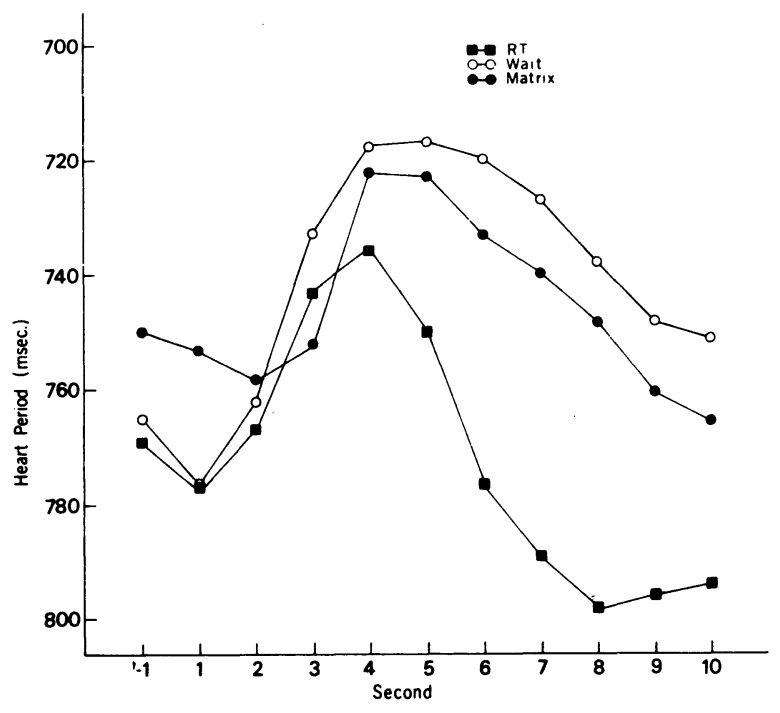

Figure 2. Second-by-second cardiac changes to task onset.

task; this was followed by an acceleratory phase $[F(10,110)=13.84, p<.01]$. Heart period during the last second of the wait subtask was maintained above pretrial level until the onset of the matrix, at which point the cardiac response showed another slight deceleration, followed by acceleration, followed by a return to base level $[F(10,100)=7.94$, $\mathrm{p}<.01]$.

The response patterns (assessed in the secondby-second analyses presented above) did not change as a function of trials in either task, although the mean heart period during the wait subtask $[F(9,99)=$ $2.87, \mathrm{p}<.01]$ and the matrix subtask $[\mathrm{F}(9,99)=$ $4.59, \mathrm{p}<.01$ ] exhibited a significant slowing as the experimental session progressed.

Analyses of trends for the three tasks, collapsed over trials, showed that, for the reaction time task, there were significant linear $[\mathrm{F}(1,11)=10.61$, $\mathrm{p}<.01]$, quadratic $[\mathrm{F}(1,11)=26.10, \mathrm{p}<.01]$, cubic $[F(1,11)=19.86, p<.01]$, and quartic $[F(1,11)=36.18, p<.01]$ components; for the wait subtask, there were significant linear $[F(1,11)=$ $7.06, \mathrm{p}<.01]$, quadratic $[\mathrm{F}(1,11)=23.53, \mathrm{p}<.01]$, and quartic $[\mathrm{F}(1,11)=52.95, \mathrm{p}<.01]$ components, while for the matrix subtask, only the quadratic $[F(1,11)=32.79, p<.01]$ and quartic $[F(1,11)=$ $20.23, p<.01]$ components were significant.

A comparison of the second-by-second cardiac response pattern across the three tasks were also made, using a 3 by 10 by 11 (Task by Trial by Second) ANOVA. In addition to a Second effect $[F(10,110)$ $=16.96, \mathrm{p}<.01]$ and a Task effect $[\mathrm{F}(2,22)=13.49$, $\mathrm{p}<.01]$, the Task by Second interaction $[\mathrm{F}(20,22)=$ $7.88, p<.01]$ confirmed that the three response patterns were statistically different. Over trials, a Trial effect $[F(9,99)=3.39, p<.01]$ and a Task by Trial interaction $[F(18,198)=2.09, p<.01]$ con- firmed differential cardiac decelerations for the three tasks as the experiment progressed.

\section{Respiratory Influences}

In order to assess the effects of respiratory changes during attentional tasks on the cardiac responses, each trial in the reaction time task as well as the wait and matrix subtasks were partitioned into three 5-sec sequential intervals: pretrial, reactive, and tonic.

For the reactive interval, mean second-by-second heart periods from the last pretrial second through the 5th second after trial onset for the five trials in which a subject showed maximum respiratory inhibition (reduced respiratory amplitude variance) were compared against the mean heart periods for the five trials in which the subject showed minimum respiratory inhibition, using 2 by 6 (Respiratory Inhibition by Second) ANOVAs.

For the tonic interval, the effects of respiratory changes were similarly assessed, using the mean heart periods and heart period $\log _{10}$ variances computed over the $5 \mathrm{sec}$ in the pretrial and in the tonic intervals as dependent measures. Separate 2 by 2 (Respiratory Inhibition by Interval) ANOVAs were used in these comparisons for each task.

Additionally, in order to ascertain that the division into maximum inhibition and minimum inhibition trials resulted in significantly different respiratory activities, respiratory amplitude $\log _{10}$ variance was compared between the reactive and the pretrial intervals, and between the tonic and pretrial intervals in separate 2 by 2 (Respiratory Inhibition by Interval) ANOVAs. Each of these analyses yielded a significant Respiratory Inhibition by Interval interaction, showing that for each task, on-task (reactive or tonic) respiratory amplitude $\log _{10}$ variance decreased from pretrial levels for the maximum inhibition trials, but increased from pretrial levels for the minimum inhibition trials. This suggests that there were meaningful differences in respiratory activity between the maximum and minimum inhibition trials. The results of the analyses on cardiac responses are summarized below and are illustrated in Table 1 and Figure 3.

\section{Reaction Time Task}

During the reactive interval of the reaction time task, respiratory inhibition affected neither the mean heart period nor the pattern of the cardiac response. During the tonic interval, heart period increased from a pretrial level of 761.8 to $789.9 \mathrm{msec}$ in the tonic interval $[\mathrm{F}(1,11)=8.86, \mathrm{p}<.025]$. In addition, a Respiratory Inhibition by Interval interaction indicated that heart period increased from 769.9 to $784.1 \mathrm{msec}$ for the minimum inhibition trials while it increased from 753.5 to $795.6 \mathrm{msec}$ for the maximum inhibition trials $[\mathrm{F}(1,11)=7.78, \mathrm{p}<.025]$. 
Table 1

Effects of Maximum and Minimum Respiratory Inhibition on Autonomic Responses

\begin{tabular}{lcccc}
\hline & \multicolumn{2}{c}{$\begin{array}{c}\text { Heart Period } \\
\text { (Milliseconds) }\end{array}$} & \multicolumn{2}{c}{$\begin{array}{c}\text { Heart Period } \\
\text { Variance }\end{array}$} \\
Task & Pretrial & Tonic & Pretrial & Tonic \\
\hline RT & & & & \\
Maximum & 753.5 & 795.6 & 2.96 & 2.83 \\
Minimum & 769.9 & 784.1 & 2.75 & 2.88 \\
Wait & & & & \\
Maximum & 756.0 & 738.0 & 2.80 & 2.89 \\
Minimum & 769.0 & 735.0 & 2.83 & 2.88 \\
Matrix & & & & \\
Maximum & 737.0 & 748.0 & 2.84 & 2.81 \\
Minimum & 747.1 & 750.3 & 2.78 & 2.71 \\
\hline
\end{tabular}

Paralleling this change, heart period $\log _{10}$ variance during the minimum inhibition trials increased from 2.75 to $2.88 \mathrm{log}$ units while it decreased from 2.96 to $2.83 \log$ units for the maximum inhibition trials $[\mathrm{F}(1,11)=11.05, \mathrm{p}<.01]$.

\section{Wait Subtask}

During the reactive interval in the wait subtask, respiratory inhibition did not affect the cardiac pattern. Likewise, during the tonic interval, no differences were found in either mean heart period or heart period $\log _{10}$ variance between the maximum and minimum inhibition trials although mean heart period during the tonic interval showed an overall decrease from $762.9 \mathrm{msec}$ during pretrial to $737.0 \mathrm{msec}$ $[F(1,11)=13.20, p<.01]$.

\section{Matrix Subtask}

In the matrix subtask, respiratory inhibition did not influence either the reactive or the tonic cardiac responses.

A comparison was made among the three tasks during the pretrial and tonic intervals, using a 3 by 2 by 2 (Task by Respiration Inhibition by Interval) ANOVA. Mean heart period exhibited a significant Task effect $[F(1,11)=20.02, p<.01]$ and Task by Interval interaction $[F(2,22)=15.12, p<.01]$, suggesting that the tonic cardiac response was task dependent. There was an overall Respiration Inhibition by Interval interaction $[F(1,11)=11.11, p<.01]$, showing that for the maximum inhibition trials there was cardiac deceleration while for the minimum inhibition trials there was a slight cardiac acceleration. For heart period $\log _{10}$ variance, the Task by Respiration Inhibition by Interval interaction was significant $[F(2,22)=3.64, p<.05]$, confirming the findings of the analyses of each individual task.

\section{DISCUSSION}

To summarize, the reactive cardiac responses seemed to be independent of the within-subject vari- ations in respiratory inhibition, while the tonic cardiac responses were linked to respiratory inhibition during the simple sensorimotor reaction time task but were independent of respiratory activity during the more cognitively mediated and difficult visual search task. These data do not contradict Hart, since his study was only concerned with the effect of pretrial respiration amplitude on phasic or reactive cardiac responses. The present study was concerned with the effects of on-task respiratory inhibition as measured by changes in respiration amplitude $\log _{10}$ variance.

A possible explanation for the differential effects may be that respiratory changes were different for the various tasks. Thus, the significant effect observed during the reaction time task might have been caused by greater respiratory changes between the minimum and maximum respiratory inhibition trials during the reaction time task than during the wait and matrix subtasks. This argument was not supported by the data. A 3 by 2 by 2 (Task by Inhibition by Interval) ANOVA comparing pretrial and tonic respiration amplitude $\log _{10}$ variance showed only a respiration inhibition effect $[\mathrm{F}(1,11)=13.78, \mathrm{p}<.01]$ and no differences among tasks.

There may be specific hypothetical explanations for the present findings. The reactive heart rate responses are often viewed as being reflexive (Sokolov, 1963). Thus, the respiratory activity might not influence the heart rate response pattern once the response is elicited. The tonic response is often associated with sustained or voluntary attention. This type of attention implies a cognitive and possibly a cortical component. As the amount of cognitive activity increases from the simple sensorimotor reaction time task to the visual search task, one may infer an increase in cortical influenes. This hypothesized increase in cortical activity may override the subcortical mechanisms responsible for respiratorycardiac coupling. This may result in the independence of the cardiac and respiratory activity during the visual search task.

The relationship between respiratory and cardiac activity during sustained attention is not a constant
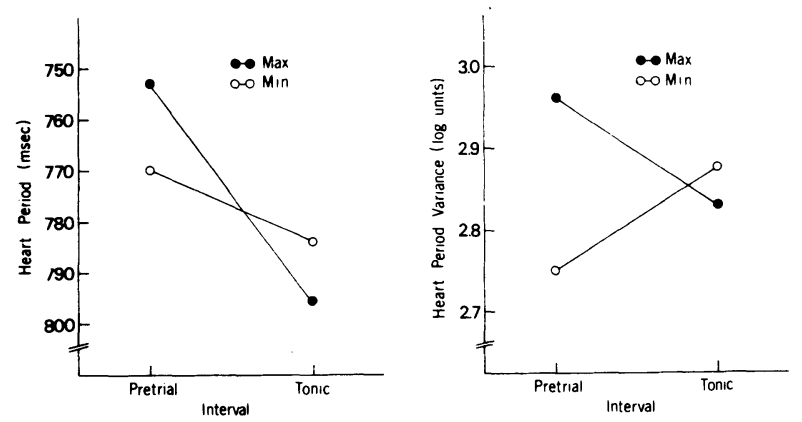

Figure 3. Tonic cardiac changes as a function of maximum and minimum respiration inhibition during the reaction time task. 
phenomenon between subjects and within the same subject. There is, however, a general trend that during sustained attention there is a reduction of heart rate variability. Thus, the simple mechanical change in respiration has a powerful influence on the cardiac response pattern. There are individual differences in the effectiveness of this relationship: Hyperactive and retarded children tend to have less coupling between respiration and cardiac response patterns than normal cohorts (see Porges, 1976). The present study adds valuable information regarding the effect of respiration on cardiac patterns by identifying task influences which affect the cardiac-respiratory relationship. The hypothesized mechanism for modulating this relationship might be the degree of cognitive activity and by inference the degree of cortical involvement in the attentional task.

\section{REFERENCE NOTE}

1. Cheung, M. N. Factor analytic components of the cardiac orienting response. In preparation.

\section{REFERENCES}

Eckoldt, K., \& Schubert, E. The influence of the tidal volume on the sinus arrhythmia of the heart. Acta Biologica et Medica Germanica, 1975, 34, 767-771.

Graham, F. K., \& Clifton, R. K. Heart rate change as a component of the orienting response. Psychological Bulletin, 1966 , 65, 305-320.

GuYton, A. C. Textbook of medical physiology (4th ed.). Philadelphia: Saunders, 1971.
HART, J. D. Cardiac response to simple stimuli as a function of phase of the respiratory cycle. Psychophysiology, 1975, 12, 634-636.

Hatton, H. M., Berg, W. K., \& Graham, F. K. Effects of acoustic rise time on heart rate response. Psychonomic Science, 1970, 19, 101-103.

Katona, P. G., \& JIH, F. Respiratory sinus arrhythmia: Noninvasive measure of parasympathetic cardiac control. Journal of Applied Physiology, 1975, 39, 301-305.

Porges, S. W. Peripheral and neurochemical parallels of psychopathology: A psychophysiological model relating autonomic imbalance to hyperactivity, psychopathy, and autism. In H. W. Reese (Ed.), Advances in child development and behavior (Vol. 1). New York: Academic Press, 1976.

Sokolov, E. N. Perception and the conditioned reflex. New York: Macmillan, 1963.

W Alter, G. F., \& Porges, S. W. Heart rate and respiratory responses as a function of task difficulty: The use of discriminant analysis in the selection of psychologically sensitive physiological responses. Psychophysiology, 1976, 13, 563-571.

\section{NOTES}

1. A relatively long rise time was used for the auditory stimulus in order to minimize acoustic startle components; see Hatton, Berg, and Graham (1970).

2 . In order to convert the beat-by-beat measures into timebased data, successive 1-sec windows were established within each trial. The weighted mean heart period for each window was computed as the sum of each heart period that occupied or partially occupied the window, multiplied by the proportion of the window that it occupied. Weighted mean respiration amplitudes were similarly computed.

(Received for publication April 25, 1976; revision accepted November 13, 1976.) 